UDK 517.538 .3

\author{
O. V. Veselovska*, V. V. Dostoina*, M. I. Klapchuk* \\ * Lviv Polytechnic National University, Lviv 79013. \\ E-mail:veselovskaov@gmail.com,v.dostoyna@gmail.com,m.klapchuk@gmail.com
}

\title{
Properties of the second-kind Chebyshev polynomials of complex variable
}

Методи розвинення функцій у ряди за системами функцій дійсної чи комплексної змінної ефективно використовують при розв'язуванні багатьох задач, у тому числі прикладного характеру. Значного розвитку набули методи розв'язання диференціальних рівнянь, що грунтуються на розвиненнях функцій у степеневі ряди, а також в ряди за системами ортогональних поліномів та інших ортогональних функцій однієї чи декількох змінних. Узагальненням методу розвинення функцій у степеневі ряди є їхнє розвинення за системою поліномів, біортогональних з деякою іншою системою функцій, які називають асоційованими. За певних умов для будь-якої незалежної $\mathrm{i}$ повної системи функцій можна побудувати відповідну систему асоційованих функцій і конструювати ряди за нею.

У даній роботі досліджено властивості поліномів Чебишова другого роду комплексної змінної. Отримано інтегральні зображення цих поліномів. Побудовано асоційовані функції, біортогональні на замкнених кривих комплексної площини з поліномами Чебишова другого роду. Встановлено достатні умови, за яких аналітичні функції можна розкласти в ряди за цією системою поліномів. Наведено приклади розкладів функцій в ряди за розглядуваною системою поліномів у комплексних областях. Крім того, отримано комбінаторні тотожності, які можна використовувати для інших досліджень.

Ключові слова: многочлени Чебишова, аналітичні функції, біортогональні системи функиій, асоиійовані функиії.

We construct a system of functions biorthogonal with Chebyshev polynomials of the second kind on closed contours in the complex plane. Properties of these functions and sufficient conditions of expansion of analytic functions into series in Chebyshev polynomials of the second kind in complex domains are investigated. The examples of such expansions are given. In addition, combinatorial identities of self-interest are obtained.

Key words: Chebyshev polynomials, analytic functions, biorthogonal systems of functions, associated functions.

MSC2010: 33E20, 33D45

\section{Introduction}

The Chebyshev polynomials form the basis of theoretical and practical studies of function approximation theory. They are effectively used in the problems of computational mathematics, for solving differential and integral equations, for numerical differentiation and integration. 
Properties of the Chebyshev polynomials of real variable are sufficiently well investigated in $[1,2]$. Considerably fewer researches concern properties of these polynomials in complex domains. Some properties of Chebyshev polynomials in a complex plane, as well as expansions of analytic functions by the Chebyshev polynomials of the first kind in complex domains are obtained in [2].

Generalization of the method of expansions of functions into power series is their expansions in system of polynomials, biorthogonal with some other system of functions which are called associated functions. Under certain conditions, for any linearly independent and complete system of functions, it is possible to construct a corresponding system of associated functions and construct series after it.

In this paper, we construct a system of functions biorthogonal with Chebyshev polynomials of the second kind on closed contours in a complex plane, and establish conditions under which analytic functions can be expanded into series in these polynomials. Examples of such expansions are given. In addition, combinatorial identities of self-interest are obtained.

Let's denote by $U_{n}(z)$ the Chebyshev polynomials of the second kind of a complex variable. For them, explicit formulas [3, p.186]

$$
U_{n}(z)=\sum_{k=0}^{\left[\frac{n}{2}\right]}(-1)^{k} 2^{n-2 k} C_{n-k}^{k} z^{n-2 k}, n=0,1, \ldots,
$$

hold. Here and below, $C_{n}^{m}$ be the binomial coefficients.

Using relations (1.1) one can obtain the expressions of the polynomials $U_{n}(z)$ for odd and even values of $n$, respectively:

$$
\begin{gathered}
U_{2 n}(z)=\sum_{k=0}^{n}(-1)^{k} 2^{2(n-k)} C_{2 n-k}^{k} z^{2(n-k)}=\sum_{l=0}^{n}(-1)^{n-l} 2^{2 l} C_{n+l}^{2 l} z^{2 l} \\
U_{2 n+1}(z)=\sum_{l=0}^{n}(-1)^{n-l} 2^{2 l+1} C_{n+l+1}^{2 l+1} z^{2 l+1} .
\end{gathered}
$$

\section{Properties of polynomials $U_{n}(z)$}

Theorem 1. The polynomials $U_{n}(z)$ can be represented as follows:

$$
U_{n}(z)=\frac{n+1}{2} \int_{-1}^{1}\left(z+t \sqrt{z^{2}-1}\right)^{n} d t .
$$

Proof. Using the Newton binomial formula and changing the order of summation, we 
find from $(2.1)$

$$
\begin{aligned}
& U_{n}(z)=\frac{n+1}{2} \sum_{m=0}^{n} C_{n}^{m} z^{n-m}\left(z^{2}-1\right)^{\frac{m}{2}} \int_{-1}^{1} t^{m} d t=(n+1) \sum_{j=0}^{\left[\frac{n}{2}\right]} \frac{C_{n}^{2 j}}{2 j+1} z^{n-2 j}\left(z^{2}-1\right)^{j}= \\
& =(n+1) \sum_{j=0}^{\left[\frac{n}{2}\right]} \frac{C_{n}^{2 j}}{2 j+1} z^{n-2 j} \sum_{k=0}^{j}(-1)^{k} C_{j}^{k} z^{2 j-2 k}=(n+1) \sum_{k=0}^{\left[\frac{n}{2}\right]}(-1)^{k} z^{n-2 k} \sum_{j=k}^{\left[\frac{n}{2}\right]} \frac{C_{n}^{2 j} C_{j}^{k}}{2 j+1} .
\end{aligned}
$$

Taking into account the combinatorial identity

$$
\sum_{j=k}^{\left[\frac{n}{2}\right]} \frac{C_{n}^{2 j} C_{j}^{k}}{2 j+1}=\frac{2^{n-2 k} C_{n-k}^{k}}{n+1}
$$

we obtain relation (1.1).

Let $\Gamma_{R}$ denote the ellipse given by the equation

$$
z=\frac{1}{2}\left(R e^{i \varphi}+R^{-1} e^{-i \varphi}\right), R>1,0 \leq \varphi<2 \pi,
$$

and $D_{R}$ be a domain whose boundary is the ellipse $\Gamma_{R}$.

Theorem 2. The polynomials $U_{n}(z)$ satisfy the following estimates:

$$
\begin{gathered}
\left|U_{n}(x)\right| \leq n+1, x \in \mathbb{R},|x| \leq 1, \\
\left|U_{n}(z)\right| \leq(n+1) R^{n}, z \in \bar{D}_{R} .
\end{gathered}
$$

Proof. Using the inequality $x^{2}-x^{2} t^{2}+t^{2} \leq 1$, which holds for all $x$ and $t$ such that $|x| \leq 1,|t| \leq 1$, from relation (2.1) it follows that

$$
\begin{gathered}
\left|U_{n}(x)\right| \leq \frac{n+1}{2} \int_{-1}^{1}\left|x+i t \sqrt{1-x^{2}}\right|^{n} d t=\frac{n+1}{2} \int_{-1}^{1}\left(x^{2}+t^{2}\left(1-x^{2}\right)\right)^{\frac{n}{2}} d t \leq \\
\leq \frac{n+1}{2} \int_{-1}^{1} d t \leq n+1 .
\end{gathered}
$$

The polynomial $\frac{1}{n+1} U_{n}(z)$ satisfies the conditions of Theorem in [4, p.166]: if for the polynomial $W_{n}(z)$ of degree $n$ on the real interval $[-1 ; 1]$ the inequality $\left|W_{n}(z)\right| \leq M$ holds, where $M=$ const, then for any $z$ outside this interval the following estimate is valid

$$
\left|W_{n}(z)\right| \leq M(a+b)^{n}
$$


where $a$ and $b$ are axes of an ellipse passing through the point $z$ with its focuses at points $z= \pm 1$. Hence, since the axes of the ellipse $\Gamma_{r}, 1<r \leq R$, with equation (2.3) are $\frac{1}{2}\left(r+\frac{1}{r}\right)$ and $\frac{1}{2}\left(r-\frac{1}{r}\right)$, respectively, then estimate (2.5) immediately follows.

Theorem 3. For the Chebyshev polynomials, integral representation

$$
U_{n}(z)=\frac{1}{2 \pi i} \int_{C}\left(z+t \sqrt{z^{2}-1}\right)^{n} \varphi_{n}(t) d t
$$

holds, where $C$ is the positively oriented circle $|t|=R_{1}, \quad 1<R_{1}<\infty$, $\varphi_{n}(t)=\frac{n+1}{2} \ln \frac{t+1}{t-1}$.

Proof. Applying the Newton binomial formula to $\left(z+t \sqrt{z^{2}-1}\right)^{n}$ and using the expansion $\varphi_{n}(t)=\sum_{j=0}^{\infty} \frac{n+1}{2 j+1} \frac{1}{t^{2 j+1}}$, according to (2.6), we obtain

$$
U_{n}(z)=(n+1) \sum_{k=0}^{n} C_{n}^{k} z^{n-k}\left(z^{2}-1\right)^{\frac{k}{2}} \sum_{j=0}^{\infty} \frac{1}{2 j+1} \frac{1}{2 \pi i} \int_{C} \frac{d t}{t^{2 j-k+1}}
$$

As usual,

$$
\delta_{m n}= \begin{cases}0, & m \neq n \\ 1, & m=n\end{cases}
$$

is the Kronecker delta.

It is known that [5, pp.81-82]

$$
\frac{1}{2 \pi i} \oint_{L} \frac{d z}{(z-a)^{n}}=\delta_{1 n}
$$

where $L$ is an arbitrary closed contour enveloping the point $a$ and once running in a positive direction. Therefore

$$
U_{n}(z)=(n+1) \sum_{j=0}^{\left[\frac{n}{2}\right]} \frac{C_{n}^{2 j}}{2 j+1} z^{n-2 j}\left(z^{2}-1\right)^{j}
$$

Using the Newton binomial formula and changing the order of summation, we have $U_{n}(z)=(n+1) \sum_{j=0}^{\left[\frac{n}{2}\right]} \frac{C_{n}^{2 j}}{2 j+1} z^{n-2 j} \sum_{k=0}^{j}(-1)^{k} C_{j}^{k} z^{2 j-2 k}=(n+1) \sum_{k=0}^{\left[\frac{n}{2}\right]}(-1)^{k} z^{n-2 k} \sum_{j=k}^{\left[\frac{n}{2}\right]} \frac{C_{n}^{2 j} C_{j}^{k}}{2 j+1}$

Hence, taking into account combinatorial identity (2.2), we obtain (1.1). 
It is known [2, p.28] that the monomial of $z^{n}$ can be uniquely expressed in terms of Chebyshev polynomials $U_{n}(z)$, as follows:

$$
z^{n}=\frac{1}{2^{n}} \sum_{k=0}^{\left[\frac{n}{2}\right]} \frac{(n-2 k+1) C_{n}^{k}}{n-k+1} U_{n-2 k}(z), n=0,1 \ldots
$$

From relations (2.8) we can obtain expressions of $z^{n}$ in the case of odd and even values of $n$, respectively:

$$
\begin{gathered}
z^{2 n}=\frac{1}{2^{2 n}} \sum_{k=0}^{n} \frac{(2 n-2 k+1) C_{2 n}^{k}}{2 n-k+1} U_{2(n-k)}(z)=\frac{1}{2^{2 n}} \sum_{j=0}^{n} \frac{(2 j+1) C_{2 n}^{n-j}}{n+j+1} U_{2 j}(z) \\
z^{2 n+1}=\frac{1}{2^{2 n+1}} \sum_{j=0}^{n} \frac{(2 j+2) C_{2 n+1}^{n-j}}{n+j+2} U_{2 j+1}(z)
\end{gathered}
$$

Theorem 4. The combinatorial identities hold:

$$
\begin{gathered}
(2 j+1) \sum_{l=j}^{n}(-1)^{n-l} \frac{C_{n+l}^{2 l} C_{2 l}^{l-j}}{l+j+1}=\delta_{n j}, \\
(2 j+2) \sum_{l=j}^{n}(-1)^{n-l} \frac{C_{n+l+1}^{2 l+1} C_{2 l+1}^{l-j}}{l+j+2}=\delta_{n j} .
\end{gathered}
$$

Proof. Substituting expressions (2.9) into (1.2) and changing the order of summation, we find that

$$
\begin{gathered}
U_{2 n}(z)=\sum_{l=0}^{n}(-1)^{n-l} C_{n+l}^{2 l} \sum_{j=0}^{l} \frac{(2 j+1) C_{2 l}^{l-j}}{j+l+1} U_{2 j}(z)= \\
=\sum_{j=0}^{n}(2 j+1) U_{2 j}(z) \sum_{l=j}^{n}(-1)^{n-l} \frac{C_{n+l}^{2 l} C_{2 l}^{l-j}}{l+j+1} .
\end{gathered}
$$

Hence, using the independence of the polynomials $U_{n}(z)$, we obtain identity (2.11). By analogy, substituting expressions (2.10) into (1.3), we obtain identity (2.12).

Let $A_{R}$ denote the space of holomorphic functions in the disk $|z|<R$, where $0<R \leq \infty$.

Theorem 5. The system of polynomials $\left\{U_{n}(z)\right\}_{n=0}^{\infty}$ is linearly independent and complete in the space $A_{R}$.

Proof. Since the coefficient of the term of the highest degree of the polynomial $U_{n}(z)$ is nonzero, then the system $\left\{U_{n}(z)\right\}_{n=0}^{\infty}$ is linearly independent [6, p.137]. In addition, it is complete [6, p.137], because every power $z^{n}$ can be uniquely expressed as a linear combination of the polynomials $U_{n}(z)$. 


\section{Functions associated with polynomials $U_{n}(z)$}

Let $f(z) \in A_{R}$. Then $f(z)$ can be represented by its Taylor series,

$$
f(z)=\sum_{n=0}^{\infty} \frac{f^{(n)}(0)}{n !} z^{n} .
$$

Find its formal expansion in system $\left\{U_{n}(z)\right\}_{n=0}^{\infty}$. Substituting relations $(2.9)$ and (2.10) into (3.1) we obtain

$$
\begin{aligned}
& f(z)=\sum_{n=0}^{\infty} \frac{f^{(2 n)}(0)}{(2 n) !} \frac{1}{2^{2 n}} \sum_{j=0}^{n} \frac{(2 j+1) C_{2 n}^{n-j}}{n+j+1} U_{2 j}(z)+ \\
& +\sum_{n=0}^{\infty} \frac{f^{(2 n+1)}(0)}{(2 n+1) !} \frac{1}{2^{2 n+1}} \sum_{j=0}^{n} \frac{(2 j+2) C_{2 n+1}^{n-j}}{n+j+2} U_{2 j+1}(z) .
\end{aligned}
$$

Changing the order of summation, we see that

$$
\begin{aligned}
& f(z)=\sum_{j=0}^{\infty}(2 j+1) U_{2 j}(z) \sum_{n=j}^{\infty} \frac{C_{2 n}^{n-j}}{2^{2 n}(n+j+1)} \frac{f^{(2 n)}(0)}{(2 n) !}+ \\
& +\sum_{j=0}^{\infty}(2 j+2) U_{2 j+1}(z) \sum_{n=j}^{\infty} \frac{C_{2 n+1}^{n-j}}{2^{2 n+1}(n+j+2)} \frac{f^{(2 n+1)}(0)}{(2 n+1) !}= \\
& =\sum_{j=0}^{\infty}(2 j+1) U_{2 j}(z) \sum_{l=0}^{\infty} \frac{C_{2(j+l)}^{l}}{2^{2 l+2 j}(l+2 j+1)} \frac{f^{(2 l+2 j)}(0)}{(2 l+2 j) !}+ \\
& +\sum_{j=0}^{\infty}(2 j+2) U_{2 j+1}(z) \sum_{l=0}^{\infty} \frac{C_{2(l+j)+1}^{l}}{2^{2 l+2 j+1}(l+2 j+2)} \frac{f^{(2 l+2 j+1)}(0)}{(2 l+2 j+1) !}= \\
& =\sum_{n=0}^{\infty}(n+1) U_{n}(z) \sum_{l=0}^{\infty} \frac{C_{2 l+n}^{l}}{2^{2 l+n}(l+n+1)} \frac{f^{(2 l+n)}(0)}{(2 l+n) !} .
\end{aligned}
$$

Introducing

$$
L_{n}(f)=(n+1) \sum_{l=0}^{\infty} \frac{C_{2 l+n}^{l}}{2^{2 l+n}(l+n+1)} \frac{f^{(2 l+n)}(0)}{(2 l+n) !}
$$

we write (3.2) in the form:

$$
f(z) \sim \sum_{n=0}^{\infty} L_{n}(f) U_{n}(z) .
$$




\section{CHEBYSHEV POLYNOMIALS OF COMPLEX VARIABLE}

By analogy with $\left[6\right.$, p.120] define the functions $\omega_{m}(z)$ associated with the polynomials $U_{n}(z)$ :

$$
\omega_{m}(z)=(m+1) \sum_{j=0}^{\infty} \frac{C_{2 j+m}^{j}}{2^{2 j+m}(j+m+1)} \frac{1}{z^{2 j+m+1}} .
$$

Using relation (3.4), expressions of the associated functions $\omega_{m}(z)$ for even and odd values of the indexes $m$ can be written as:

$$
\begin{gathered}
\omega_{2 m}(z)=(2 m+1) \sum_{j=0}^{\infty} \frac{C_{2 j+2 m}^{j}}{2^{2 j+2 m}(j+2 m+1)} \frac{1}{z^{2 j+2 m+1}}=(2 m+1) \sum_{l=m}^{\infty} \frac{C_{2 l}^{l-m}}{2^{2 l}(l+m+1)} \frac{1}{z^{2 l+1}}, \\
\omega_{2 m+1}(z)=(2 m+2) \sum_{l=m}^{\infty} \frac{C_{2 l+1}^{l-m}}{2^{2 l+1}(l+m+2)} \frac{1}{z^{2 l+2}}
\end{gathered}
$$

Theorem 6. The functions $\omega_{m}(z)$ are analytic in the domain $|z|>1$. Then, the coefficients $L_{m}(f)$ can be written in the integral form

$$
L_{m}(f)=\frac{1}{2 \pi i} \int_{C} f(z) \omega_{m}(z) d z
$$

where $C$ is positively oriented circle $|z|=q, 1<q<R$.

Proof. From the asymptotical formula $n ! \sim \frac{n^{n}}{e^{n}}, n \rightarrow \infty$, it follows that

$$
\begin{aligned}
& \frac{C_{2 j+m}^{j}}{2^{2 j+m}(j+m+1)}=\frac{(2 j+m) !}{2^{2 j+m}(j+m+1) j !(j+m) !} \sim \frac{(2 j+m)^{2 j+m}}{2^{2 j+m}(j+m+1) j^{j}(j+m)^{j+m}}= \\
& =\frac{(2 j)^{2 j+m}\left(1+\frac{m}{2 j}\right)^{2 j+m}}{2^{2 j+m}(j+m+1) j^{2 j+m}\left(1+\frac{m}{j}\right)^{j+m}}=\frac{\left(1+\frac{m}{2 j}\right)^{2 j+m}}{(j+m+1)\left(1+\frac{m}{j}\right)^{j+m}} \sim \frac{1}{j+m+1},
\end{aligned}
$$

where $j \rightarrow \infty$.

Since for a fixed value of $m$ the limit

$$
\lim _{j \rightarrow \infty} \sqrt[j]{\frac{(m+1) C_{2 j+m}^{j}}{2^{2 j+m}(j+m+1)}}=\lim _{j \rightarrow \infty} \sqrt[j]{\frac{m+1}{j+m+1}}=1
$$

exists, then the series in (3.4) converges and represents an analytic function in the domain $|z|>1$. 
Definition 1. A system $\left\{\omega_{k}(z)\right\}_{k=0}^{\infty}$ of associated functions is called biorthogonal to a system of polynomials $V_{n}(z)$ if

$$
\frac{1}{2 \pi i} \int_{L} V_{n}(z) \omega_{k}(z) d z=\delta_{n k}
$$

where $L$ is a closed positively oriented contour containing the singular points of functions $\omega_{k}(z)$.

Theorem 7. The system of associated functions $\left\{\omega_{n}(z)\right\}_{n=0}^{\infty}$ is biorthogonal to the system of polynomials $U_{n}(z)$ along any piecewise-smooth closed contour $\gamma$ enveloping the disk $|z| \leq 1$, i. e.

$$
\frac{1}{2 \pi i} \int_{\gamma} U_{n}(z) \omega_{m}(z) d z=\delta_{n m}
$$

Proof. First, we consider the case of even values of indices $n$ and $m$ in formula (3.9). Substituting expressions (1.2) and (3.5) into the left-hand side of (3.9), we obtain

$$
\frac{1}{2 \pi i} \int_{\gamma} U_{2 n}(z) \omega_{2 m}(z) d z=\sum_{j=0}^{n}(-1)^{n-j} 2^{2 j} C_{n+j}^{2 j}(2 m+1) \sum_{l=m}^{\infty} \frac{C_{2 l}^{l-m}}{2^{2 l}(l+m+1)} \frac{1}{2 \pi i} \int_{\gamma} \frac{d z}{z^{2(l-j)+1}} .
$$

Hence, accordingly with (2.7), we have

$$
\frac{1}{2 \pi i} \int_{\gamma} U_{2 n}(z) \omega_{2 m}(z) d z=(2 m+1) \sum_{j=m}^{n}(-1)^{n-j} \frac{C_{n+j}^{2 j} C_{2 j}^{j-m}}{j+m+1} .
$$

Similarly, we find for odd values of indices $m$ and $n$ :

$$
\frac{1}{2 \pi i} \int_{\gamma} U_{2 n+1}(z) \omega_{2 m+1}(z) d z=(2 m+2) \sum_{j=m}^{n}(-1)^{n-j} \frac{C_{n+j+1}^{2 j+1} C_{2 j+1}^{j-m}}{j+m+2} .
$$

Using combinatorial identities (2.11) and (2.12), we obtain equality (3.9).

The equalities

$$
\frac{1}{2 \pi i} \int_{\gamma} U_{2 n+1}(z) \omega_{2 m}(z) d z=0, \frac{1}{2 \pi i} \int_{\gamma} U_{2 n}(z) \omega_{2 m+1}(z) d z=0
$$

are obvious because there are no terms of $z^{-1}$.

Theorem 8. The associated functions $\omega_{n}(z)$ can be represented in the integral form

$$
\omega_{n}(z)=\frac{2}{\pi} \int_{-1}^{1} \frac{\sqrt{1-x^{2}}}{z-x} U_{n}(x) d x
$$


Proof. For the polynomials $U_{n}(x)$, let us write the Cauchy integral formula,

$$
U_{n}(x)=\frac{1}{2 \pi i} \int_{\gamma} \frac{U_{n}(z)}{z-x} d z
$$

where $\gamma$ is the closed contour that is the boundary of a domain containing the interval $[-1 ; 1]$. The conditions of orthogonality [2, p.52] for the polynomials $U_{n}(x)$ has the form:

$$
\frac{2}{\pi} \int_{-1}^{1} U_{n}(x) U_{m}(x) \sqrt{1-x^{2}} d x=\delta_{n m} .
$$

Substituting (3.11) into (3.12), we obtain

$$
\frac{2}{\pi} \int_{-1}^{1}\left(\frac{1}{2 \pi i} \int_{\gamma} \frac{U_{n}(z)}{z-x} d z\right) U_{m}(x) \sqrt{1-x^{2}} d x=\delta_{n m} .
$$

Changing the order of integration in the left-hand side of the last equality, we find

$$
\frac{1}{2 \pi i} \int_{\gamma} U_{n}(z) \frac{2}{\pi}\left(\int_{-1}^{1} \frac{U_{m}(x) \sqrt{1-x^{2}}}{z-x} d x\right) d z=\delta_{n m} .
$$

Hence, by means of (3.9), we obtain integral representation (3.10).

Theorem 9. For the associated functions $\omega_{n}(z)$ the following inequalities hold:

$$
\left|\omega_{n}(z)\right| \leq \frac{n+1}{\rho^{n}(\rho-1)}
$$

for $|z| \geq \rho, \rho>1$;

$$
\left|\omega_{n}(z)\right| \leq \frac{4(n+1)}{R^{n-1}(R-1)}
$$

for $z \in \Gamma_{R}, R>1$, where $\Gamma_{R}$ is the ellipse given by (2.3).

Proof. Substituting the expansion

$$
\frac{1}{z-x}=\sum_{k=0}^{\infty} \frac{x^{k}}{z^{k+1}},|z|>1
$$

into (3.10) and taking into account the equality

$$
\int_{-1}^{1} x^{k} \sqrt{1-x^{2}} U_{n}(x) d x=0,0 \leq k<n
$$


we obtain

$$
\omega_{n}(z)=\frac{2}{\pi} \sum_{k=n}^{\infty} \frac{1}{z^{k+1}} \int_{-1}^{1} x^{k} \sqrt{1-x^{2}} U_{n}(x) d x .
$$

By estimate (2.4) we find that

$$
\left|\omega_{n}(z)\right| \leq \frac{2}{\pi} \sum_{k=n}^{\infty} \frac{1}{|z|^{k+1}} \int_{-1}^{1}|x|^{k} \sqrt{1-x^{2}}\left|U_{n}(x)\right| d x \leq \frac{2(n+1)}{\pi} \sum_{k=n}^{\infty} \frac{1}{|z|^{k+1}} \int_{-1}^{1} \sqrt{1-x^{2}} d x .
$$

Since

$$
\int_{-1}^{1} \sqrt{1-x^{2}} d x=\int_{-\pi / 2}^{\pi / 2} \cos ^{2} t d t=\frac{\pi}{2}
$$

then

$$
\left|\omega_{n}(z)\right| \leq(n+1) \sum_{k=n}^{\infty} \frac{1}{|z|^{k+1}} \leq \frac{n+1}{\rho^{n}(\rho-1)} .
$$

It is known [2, p.149] that

$$
\frac{1}{z-x}=\frac{4 p}{p^{2}-1} \sum_{k=0}^{\infty} \frac{T_{k}(x)}{p^{k}}
$$

where $T_{k}(x)$ is the $k$-th degree Chebyshev polynomial of the first kind;

$$
\begin{gathered}
p=z+\sqrt{z^{2}-1} ; \\
\sum_{k=0}^{\infty} a_{k} T_{k}(x)=\frac{a_{0}}{2} T_{0}(x)+a_{1} T_{1}(x)+\ldots+a_{n} T_{n}(x)+\ldots
\end{gathered}
$$

If we substitute (3.16) into (3.10) and take into account the equality

$$
\int_{-1}^{1} \sqrt{1-x^{2}} U_{n}(x) T_{k}(x) d x=0, k<n
$$

we obtain

$$
\omega_{n}(z)=\frac{8 p}{\pi\left(p^{2}-1\right)} \sum_{k=n}^{\infty} \frac{1}{p^{k}} \int_{-1}^{1} \sqrt{1-x^{2}} U_{n}(x) T_{k}(x) d x .
$$

Using the inequality [2, p.46] $\left|T_{k}(x)\right| \leq 1, k=0,1, \ldots$, which holds for real $x$ such that $|x| \leq 1$, estimate (2.5), and equality (3.15), we obtain

$$
\left|\omega_{n}(z)\right| \leq \frac{8|p|}{\pi\left|p^{2}-1\right|} \sum_{k=n}^{\infty} \frac{1}{\left|p^{k}\right|} \int_{-1}^{1} \sqrt{1-x^{2}}\left|U_{n}(x)\right|\left|T_{k}(x)\right| d x \leq
$$




$$
\leq \frac{8|p|(n+1)}{\pi\left|p^{2}-1\right|} \sum_{k=n}^{\infty} \frac{1}{\left|p^{k}\right|} \int_{-1}^{1} \sqrt{1-x^{2}} d x=\frac{4|p|(n+1)}{\left|p^{2}-1\right|} \sum_{k=n}^{\infty} \frac{1}{\left|p^{k}\right|} \leq \frac{4(n+1)}{R^{n-1}(R-1)}
$$

Theorem 10. The expansion

$$
\frac{1}{t-z}=\sum_{n=0}^{\infty} U_{n}(z) \omega_{n}(t)
$$

is holds. Furthermore, the series in (3.17) converges uniformly for $t \in \bar{D}_{\rho}^{\infty}, z \in \bar{D}_{r}^{0}$, where $\rho$ and $r$ are numbers such that $0<r<\infty, \rho>\max \{1, r\}, \bar{D}_{\rho}^{\infty}$ is the closed domain containing the infinity with boundary $\Gamma_{\rho}, \bar{D}_{r}^{0}$ is the closed domain containing zero with boundary $\Gamma_{r}$.

Proof. Substituting expressions (3.5) and (3.6) into the right-hand side of expansion (3.17), we obtain

$$
\begin{gathered}
\sum_{n=0}^{\infty} U_{n}(z) \omega_{n}(t)=\sum_{n=0}^{\infty}(2 n+1) U_{2 n}(z) \sum_{l=n}^{\infty} \frac{C_{2 l}^{l-n}}{2^{2 l}(l+n+1)} \frac{1}{t^{2 l+1}}+ \\
\quad+\sum_{n=0}^{\infty}(2 n+2) U_{2 n+1}(z) \sum_{l=n}^{\infty} \frac{C_{2 l+1}^{l-n}}{2^{2 l+1}(l+n+2)} \frac{1}{t^{2 l+2}}
\end{gathered}
$$

Changing the order of summation in the last two sums and taking into account (2.9) and (2.10), we find that

$$
\begin{gathered}
\sum_{n=0}^{\infty} U_{n}(z) \omega_{n}(t)=\sum_{l=0}^{\infty} \frac{1}{t^{2 l+1}} \frac{1}{2^{2 l}} \sum_{n=0}^{l} \frac{(2 n+1) C_{2 l}^{l-n}}{n+l+1} U_{2 n}(z)+ \\
+\sum_{l=0}^{\infty} \frac{1}{t^{2 l+2}} \frac{1}{2^{2 l+1}} \sum_{n=0}^{l} \frac{(2 n+2) C_{2 l+1}^{l-n}}{n+l+2} U_{2 n+1}(z)=\sum_{l=0}^{\infty} \frac{z^{2 l}}{t^{2 l+1}}+\sum_{l=0}^{\infty} \frac{z^{2 l+1}}{t^{2 l+2}}=\frac{1}{t} \sum_{m=0}^{\infty}\left(\frac{z}{t}\right)^{m}= \\
=\frac{1}{t} \frac{1}{1-\frac{z}{t}}=\frac{1}{t-z} .
\end{gathered}
$$

Now, let us show that the series in (3.17) is uniformly convergent for $t \in \bar{D}_{\rho}^{\infty}$, $z \in \bar{D}_{r}^{0}$. Here $\rho$ and $r$ are numbers such that $0<r<\infty, \rho>\max \{1, r\}$.

On the basis of (2.5) and (3.13), we have

$$
\left|\sum_{n=0}^{\infty} U_{n}(z) \omega_{n}(t)\right| \leq \sum_{n=0}^{\infty}\left|U_{n}(z)\right|\left|\omega_{n}(t)\right| \leq \frac{1}{\rho-1} \sum_{n=0}^{\infty}(n+1)^{2}\left(\frac{r}{\rho}\right)^{n} .
$$

Since $\rho>r$, the above series converges. Therefore, the series in (3.17) converges uniformly in the above mentioned domains. 


\section{Expansion of functions into series in polynomials $U_{n}(z)$}

Theorem 11. Let $f(z)$ be a function of complex variable which is holomorphic in an open domain $D_{R}$ whose boundary is ellipse $\Gamma_{R}, 1<R \leq \infty$, with equation (2.3) and bounded by $M$ in $\Gamma_{R}$, i. e.

$$
|f(z)| \leq M, z \in \Gamma_{R}
$$

where $M=$ const.

Then the series

$$
\sum_{n=0}^{\infty} L_{n}(f) U_{n}(z)
$$

converges uniformly in the closed domain $\bar{D}_{r}$ whose boundary is the ellipse $\Gamma_{r}$, where $1 \leq r<R$.

Here $L_{n}(f)$ are the coefficients defined by (3.3).

Proof. Replacing $C$ by $\Gamma_{R}$ in (3.7) and using (4.1) and (3.14), we estimate the coefficients $L_{n}(f)$ :

$$
\left|L_{n}(f)\right| \leq \frac{1}{2 \pi} \int_{\Gamma_{R}}|f(z)|\left|\omega_{n}(z)\right| d z \leq \frac{4 M(n+1)}{R^{n-1}(R-1)} \int_{\Gamma_{R}} d z .
$$

Since $\Gamma_{R}$ is an ellipse with axes $2 a=R+\frac{1}{R}, 2 b=R-\frac{1}{R}$ for $R>1$, we see that

$$
\begin{gathered}
\int_{\Gamma_{R}} d z=4 \int_{0}^{\frac{\pi}{2}} \sqrt{a^{2} \sin ^{2} \varphi+b^{2} \cos ^{2} \varphi} d \varphi=2 \int_{0}^{\frac{\pi}{2}} \sqrt{R^{2}+\frac{1}{R^{2}}-2 \cos 2 \varphi} d \varphi \leq \\
\leq 2 \int_{0}^{\frac{\pi}{2}}\left(R+\frac{1}{R}\right) d \varphi=\frac{\pi\left(R^{2}+1\right)}{R}
\end{gathered}
$$

and

$$
\left|L_{n}(f)\right| \leq \frac{B(n+1)\left(R^{2}+1\right)}{R^{n}}, B=\text { const. }
$$

Now, according to (2.5), we obtain

$$
\left|\sum_{n=0}^{\infty} L_{n}(f) U_{n}(z)\right| \leq \sum_{n=0}^{\infty}\left|L_{n}(f)\right|\left|U_{n}(z)\right| \leq \sum_{n=0}^{\infty}(n+1)^{2}\left(\frac{r}{R}\right)^{n},
$$

where $z \in \bar{D}_{r}$. The last series converges for $r<R$. Therefore, the series in (4.2) converges uniformly in the closed domain $\bar{D}_{r}$. 


\section{CHEBYSHEV POLYNOMIALS OF COMPLEX VARIABLE}

Let $a$ be a complex constant ( $a=$ const).

Example 1. Expand the function $f(z)=\frac{1}{a-z}$ into series in polynomials $U_{n}(z)$. Using expansion (3.17), we obtain

$$
\frac{1}{a-z}=\sum_{n=0}^{\infty} \omega_{n}(a) U_{n}(z)(|a|>|z|)
$$

where $\omega_{n}(z)$ are the associated functions defined by (3.4).

Example 2. Expand the function $f(z)=\frac{a}{a^{2}-z^{2}}$ into series in polynomials $U_{n}(z)$.

It is known [2, p.13]

$$
U_{n}(-z)=(-1)^{n} U_{n}(z)
$$

From the relation

$$
\frac{1}{a^{2}-z^{2}}=\frac{1}{2 a}\left(\frac{1}{a-z}+\frac{1}{a+z}\right),
$$

example 1 , and equation (4.3), we obtain

$$
\frac{a}{a^{2}-z^{2}}=\frac{1}{2}\left(\sum_{n=0}^{\infty} \omega_{n}(a) U_{n}(z)+\sum_{n=0}^{\infty}(-1)^{n} \omega_{n}(a) U_{n}(z)\right)=\frac{1}{2} \sum_{n=0}^{\infty}\left(1+(-1)^{n}\right) \omega_{n}(a) U_{n}(z) .
$$

From here

$$
\frac{a}{a^{2}-z^{2}}=\sum_{k=0}^{\infty} \omega_{2 k}(a) U_{2 k}(z)(|a|>|z|) .
$$

Example 3. Expand the function $\frac{z}{a^{2}-z^{2}}$ into series in polynomials $U_{n}(z)$. Using the relation

$$
\frac{z}{a^{2}-z^{2}}=\frac{a+z-a}{a^{2}-z^{2}}=\frac{1}{a-z}-\frac{a}{a^{2}-z^{2}},
$$

examples 1 , and 2 , we obtain

$$
\frac{z}{a^{2}-z^{2}}=\sum_{n=0}^{\infty} \omega_{n}(a) U_{n}(z)-\frac{1}{2} \sum_{n=0}^{\infty}\left(1+(-1)^{n}\right) \omega_{n}(a) U_{n}(z)=\frac{1}{2} \sum_{n=0}^{\infty}\left(1-(-1)^{n}\right) \omega_{n}(a) U_{n}(z) .
$$

Hence

$$
\frac{z}{a^{2}-z^{2}}=\sum_{k=0}^{\infty} \omega_{2 k+1}(a) U_{2 k+1}(z)(|a|>|z|) .
$$

Example 4. Expand the function $f(z)=e^{a z}$ into series in polynomials $U_{n}(z)$. Since $f^{(s)}(0)=a^{s}$, then from relation (3.3) we find

$$
\begin{aligned}
L_{n}(f)= & \sum_{j=0}^{\infty} \frac{(n+1) C_{2 j+n}^{j}}{2^{2 j+n}(j+n+1)} \frac{a^{2 j+n}}{(2 j+n) !}=\sum_{j=0}^{\infty} \frac{(n+1)}{j !(j+n+1) !}\left(\frac{a}{2}\right)^{2 j+n}= \\
& =\frac{2}{a} \sum_{j=0}^{\infty} \frac{(n+1)}{j !(j+n+1) !}\left(\frac{a}{2}\right)^{2 j+n+1}=\frac{2(n+1)}{a} I_{n+1}(a),
\end{aligned}
$$


where $I_{\nu}(z)=\sum_{j=0}^{\infty} \frac{1}{j ! \Gamma(j+\nu+1)}\left(\frac{z}{2}\right)^{2 j+\nu}$ are modified Bessel functions of the first kind [3, p.13]. Therefore

$$
e^{a z}=\frac{2}{a} \sum_{n=0}^{\infty}(n+1) I_{n+1}(a) U_{n}(z) .
$$

From (4.4), taking into account equality (4.3) and using the relation

$$
I_{n}(i a)=i^{n} J_{n}(a)
$$

where $J_{\nu}(z)=\sum_{j=0}^{\infty} \frac{(-1)^{j}}{j ! \Gamma(j+\nu+1)}\left(\frac{z}{2}\right)^{2 j+\nu}$ are the Bessel functions of the first kind $[3$, p.12], we obtain the following expansions:

$$
\begin{gathered}
e^{-a z}=\frac{2}{a} \sum_{n=0}^{\infty}(-1)^{n}(n+1) I_{n+1}(a) U_{n}(z), \\
e^{i a z}=\frac{2}{a} \sum_{n=0}^{\infty} i^{n}(n+1) J_{n+1}(a) U_{n}(z), \\
e^{-i a z}=\frac{2}{a} \sum_{n=0}^{\infty}(-1)^{n}(n+1) i^{n} J_{n+1}(a) U_{n}(z) .
\end{gathered}
$$

From relations (4.4)-(4.7), the expansions of trigonometrical and hyperbolic functions in polynomials $U_{n}(z)$ can be obtain.

Example 5. Expand the function $f(z)=\sin a z$ into series in polynomials $U_{n}(z)$.

Since $\sin a z=\frac{1}{2 i}\left(e^{i a z}-e^{-i a z}\right)$, then, taking into account expansions (4.6) and (4.7), we have

$$
\begin{aligned}
\sin a z= & \frac{1}{i a} \sum_{n=0}^{\infty}\left(1-(-1)^{n}\right)(n+1) i^{n} J_{n+1}(a) U_{n}(z)= \\
& \frac{2}{a} \sum_{k=0}^{\infty}(-1)^{k}(2 k+2) J_{2 k+2}(a) U_{2 k+1}(z) .
\end{aligned}
$$

Example 6. Expand the function $f(z)=\cos a z$ into series in polynomials $U_{n}(z)$. From (4.6) and (4.7) it follows

$$
\begin{aligned}
\cos a z=\frac{1}{2}\left(e^{i a z}\right. & \left.+e^{-i a z}\right)=\frac{1}{a} \sum_{n=0}^{\infty}\left(1+(-1)^{n}\right)(n+1) i^{n} J_{n+1}(a) U_{n}(z)= \\
& =\frac{2}{a} \sum_{k=0}^{\infty}(-1)^{k}(2 k+1) J_{2 k+1}(a) U_{2 k}(z)
\end{aligned}
$$


Example 7. Expand the function $f(z)=\operatorname{sh} a z$ into series in polynomials $U_{n}(z)$. Since $\operatorname{sh} a z=\frac{1}{2}\left(e^{a z}-e^{-a z}\right)$, then $\operatorname{sh} a z=\frac{1}{a} \sum_{n=0}^{\infty}\left(1-(-1)^{n}\right)(n+1) I_{n+1}(a) U_{n}(z)=\frac{2}{a} \sum_{k=0}^{\infty}(2 k+2) I_{2 k+2}(a) U_{2 k+1}(z)$.

Example 8. Expand the function $f(z)=$ ch $a z$ into series in polynomials $U_{n}(z)$. We have

$$
\begin{gathered}
\operatorname{ch} a z=\frac{1}{2}\left(e^{a z}+e^{-a z}\right)=\frac{1}{a} \sum_{n=0}^{\infty}\left(1+(-1)^{n}\right)(n+1) I_{n+1}(a) U_{n}(z)= \\
=\frac{2}{a} \sum_{k=0}^{\infty}(2 k+1) I_{2 k+1}(a) U_{2 k}(z) .
\end{gathered}
$$

\section{Conclusions}

Methods for expanding functions into series in systems of functions of a real or complex variable are effectively used for the construction of solutions of boundary value problems for ordinary or partial differential equations. Methods of solving differential equations, which are based on the expansion of functions in power series, as well as into series in systems of orthogonal polynomials and other orthogonal functions of one and several variables have gained considerable development. The systems of biorthogonal functions represent wider class, than orthogonal functions. Determination of series coefficients is based on the property of biorthogonality. They are expressed via the derivatives of the functions that are expanded in these series.

In this paper, we construct the associated functions with Chebyshev polynomials of the second kind biorthogonal on closed curves of a complex plane. Conditions under which the analytic functions can be expanded into series in this system are established. Examples of such functions series in the considered system of polynomials are given. In addition, combinatorial identities of self-interest are obtained. Results of this article can be useful in several physics and engineering problems, for instance, in electromagnetic field problems $[7,8]$.

\section{References}

1. Szegö G.: Orthogonal polynomials. Amer. Math. Soc. Colloq. Publ. vol. XXIII, Amer. Math. Soc, New York, 1959; Russian transl. in: Fizmatgiz, Moscow, (1962).

2. Pashkovskij S.: Vychislitel'nye primenenija mnogochlenov i rjadov Chebyshova. Moscow, Nauka, (1983). (in Russian)

3. Bateman H.: Higher Transcendental Functions, vols. I and II. Based, in part, on notes left by Harry Bateman. Ed. McGraw-Hill, New York-London, (1953). doi: 10.1126/science.120.3112.302-b 
4. Polya G., Szegő G.: Problems and Theorems in Analysis II Theory of Functions. Zeros. Polynomials. Determinants. Number Theory. Geometry, Springer-Verlag, Berlin, Heidelberg, (1998) doi.org/10.1007/978-3-642-61905-2

5. Zheverzheev V., Kal'nickij L., Sapogov N.: Special'nyj kurs vysshej matematiki dlja vtuzov. Moscow, Vysshaja shkola, (1970). (in Russian)

6. Markushevich A.: Theory of functions of a complex variable; rev. English ed. translated and edited by Richard A. Silverman Chelsea Pub. Co., New York, (1977).

7. Assante D., Davino D. Falco S., Schettino F., Verolino L.: Coupling impedance of a charge traveling in a drift tube. IEEE Transactions on Magnetics, 41(5) (2005), 1924-1927. doi: 10.1109/TMAG. 2005.846226

8. Assante D., Verolino L.: Efficient evaluation of the longitudinal coupling impedance of a plane strip. Progress in Electromagnetics Research M, 26 (2012), 251-265. doi: 10.2528/PIERM12091309

Received: 07.05.2020. Accepted: 14.11.2020 